\title{
Symptom Persistence after Iron Normalization in Women with Restless Legs Syndrome
}

\author{
Young Rong Bang ${ }^{1}$, Hong Jun Jeon ${ }^{2}$, Hye Youn Park', and In-Young Yoon ${ }^{1,3 凶}$ \\ ${ }^{1}$ Department of Neuropsychiatry, Seoul National University Bundang Hospital, Seongnam, Republic of Korea \\ ${ }^{2}$ Department of Psychiatry, Konkuk University Medical Center, Seoul, Republic of Korea \\ ${ }^{3}$ Department of Psychiatry, Seoul National University College of Medicine, Seoul, Republic of Korea
}

\begin{abstract}
Objective The aim of this study was to determine the clinical course of restless legs syndrome (RLS) and potential risk factors for the persistence of RLS symptoms after iron normalization in women with RLS and low serum ferritin $(<50 \mu \mathrm{g} / \mathrm{L})$.

Methods We reviewed 39 women with RLS and iron deficiency, who achieved iron normalization after oral iron replacement for three months. Risk factors contributing to symptom persistence were estimated by logistic regression analyses. Remission was defined as no RLS symptoms for at least 6 months after the iron normalization.

Results Over the observation period of $2.5 \pm 1.4$ years, 15 patients reported no RLS symptom whereas 24 patients still complained of RLS symptoms. The remission rate of RLS with iron replacement was 38.5\%. The relative risk of symptom persistence was increased by the duration of RLS symptoms (OR: 1.88, 95\% CI: 1.01-3.49) or by the age at RLS diagnosis (OR: 1.25, 95\% CI: 1.01-1.56).

Conclusion Almost two-third of RLS patients with iron deficiency showed persistence of the symptom even after iron normalization. Considering that longer duration of RLS symptoms and older age at RLS diagnosis were risk factors for symptom persistence, early intervention of iron deficiency in RLS is warranted.

Psychiatry Investig 2018;15(4):390-395
\end{abstract}

Key Words Ferritin, Iron deficiency, Persistence, Restless legs syndrome, Risk factor.

\section{INTRODUCTION}

Restless legs syndrome (RLS) is an urge to move the legs, usually accompanied by uncomfortable and unpleasant sensation in the legs. It is associated with disrupted sleep and lower quality of life. ${ }^{1}$ The clinical course of RLS is known to fluctuate throughout a patient's life. However, it has been recently reported that patients with greater RLS severity are more likely to have a chronic course. ${ }^{2}$ RLS can be divided into primary or idiopathic and secondary forms. Well-documented secondary cause of RLS is an iron deficiency condition including end-stage renal disease, pregnancy, iron deficiency anemia and gastric surgery. ${ }^{3}$ Although the pathogenesis of RLS is un-

Received: May 10, 2017 Revised: August 16, 2017

Accepted: August 21, 2017

$\triangle$ Correspondence: In-Young Yoon, MD, PhD

Department of Neuropsychiatry, Seoul National University Bundang Hospital, 82 Gumi-ro 173beon-gil, Bundang-gu, Seongnam 13620, Republic of Korea Tel: +82-31-787-7433, Fax: +82-31-787-4058, E-mail: iyoon@snu.ac.kr

(c) This is an Open Access article distributed under the terms of the Creative Commons Attribution Non-Commercial License (http://creativecommons.org/licenses/bync/4.0) which permits unrestricted non-commercial use, distribution, and reproduction in any medium, provided the original work is properly cited. known, the abnormality of dopamine system is suggested as a mechanism for developing RLS and therapeutic effects of dopamine agonists have been clinically demonstrated. On the other hand, iron supplementation has been first recommended for RLS patients with low serum ferritin level $(50 \mathrm{ng} / \mathrm{mL}$ or less) according to management guidelines. ${ }^{4,5}$

Two randomized, placebo-controlled studies have documented that iron replacement treatment was beneficial for RLS patients with low serum ferritin level. ${ }^{6,7}$ Wang et al. have compared the efficacy of oral iron therapy in eighteen participants, and found that symptom severity measured by the International RLS study group rating scale for severity (IRLS) ${ }^{8}$ scores was decreased in the treatment group compared to that in the placebo group after 12 weeks of therapy. ${ }^{6}$ The mean IRLS score change in the treatment group, was greater than that in the placebo group. The response rate defined as improvement of quality of life was reported to be $63.6 \%$. Relatively small sample size and short follow-up period to estimate the efficacy of iron treatment remained as limitations of the study. Grote et $\mathrm{al}^{7}$ have performed a study with sixty RLS patients from multi-centers and prospectively followed-up the effica- 
cy of intravenous (IV) iron sucrose compared to placebo until 12 months. The response rate which was defined as $\geq 50 \%$ reduction of IRLS scores at 11 weeks after iron treatment was $65 \%$. Since oral iron is widely used in the treatment of iron deficiency, outcomes including efficacy and side effects of IV iron sucrose may be limited when generalized in real clinical settings. In our recent randomized controlled trial comparing oral iron therapy and pramipexole, we observed a modest response rate of $47 \%$ to iron replacement for fifteen RLS patients with low serum ferritin levels. ${ }^{9}$ Since it was not a naturalistic study, little was known about the clinical course of RLS after correcting iron deficiency by oral iron treatment.

Although the response of iron treatment has been confirmed in RLS patients with low serum ferritin levels compared to placebo, not all RLS patients with low serum ferritin have shown sufficient response to iron treatment. The factors contributing to the persistence of RLS symptoms after the iron treatment are currently unknown. Up to date, the course and prognosis after iron treatment in patients of RLS with low serum ferritin levels have not been reported yet. Therefore, the objective of this current study was to extend the knowledge of the clinical course of RLS and potential risk factors associated with symptom persistence after iron replacement in RLS patients with low serum ferritin levels.

\section{METHODS}

\section{Subjects}

For retrospective analysis, female patients with RLS confirmed as iron deficiency at first visit were selected from the chart of the sleep clinic of Seoul National University Bundang Hospital (SNUBH) from January 2008 to December 2014. All patients were diagnosed as RLS by two sleep experts based on the essential criteria for RLS recommended by the National Institutes of Health (NIH). ${ }^{10}$ To exclude RLS mim$\mathrm{ics}^{11}$ such as myalgia, venous stasis, leg edema, arthritis, leg cramps, and positional discomfort, we used additional clinical examination and mimics-related questions. According to RLS treatment guidelines, ${ }^{4,5}$ the oral iron therapy was applied to the patients with serum ferritin concentration lower than $50 \mu \mathrm{g} / \mathrm{L}$ or iron saturation lower than $17 \%$ at their first visit. Patients were recommended to take oral iron supplement of iron sulfate $256 \mathrm{mg}$ with ascorbic acid $30 \mathrm{mg}$ twice a day for three months as the guidelines. If the patients complained of remaining symptom even after iron normalization, they were given additional medications such as dopamine agonists or $\alpha 2 \delta$ ligand. Only subjects who achieved ferritin concentration $\geq 50 \mu \mathrm{g} / \mathrm{L}$ or iron saturation $>20 \%$ and were observed for a period of at least 6 months after correcting serum ferritin were selected. Drop-outs with no follow-up data of serum ferritin or incomplete iron replacement, and individuals who had comorbidities including pregnancy, end-stage renal disease, malignancy, and other medical diseases causing iron deficiency were excluded from this study. The study protocol was approved by the Institutional Review Board of SNUBH (B-1606-350-102).

\section{Baseline assessments}

Baseline demographic and laboratory data at the initial diagnosis were extracted from electronic medical records of patients. All subjects were routinely evaluated for RLS severity using IRLS at the first visit. Participants were classified into the following four groups based on total IRLS scores for RLS severity; mild (1-10), moderate (11-20), severe (21-30), and very severe (31-40). In addition, we asked patients about several characteristics of RLS, including symmetry, presence of upper limb symptoms or pain, and family history. The other information of several questionnaires including the Pittsburgh sleep quality index (PSQI) for subjective sleep complaints, ${ }^{12}$ the Epworth sleepiness scale (ESS) for daytime sleepiness, ${ }^{13}$ and the Beck depression inventory (BDI) for depressive symptoms were also obtained. In addition, based on medical records or interviews, we determined whether patients had been taking medications such as antidepressants known to generate or exacerbate RLS symptoms.

\section{Follow-up procedures and outcomes}

Follow-up evaluation of RLS symptom at 6 months or more after achieving iron normalization had been conducted in June 2015 by face-to-face or telephone interviews as a part of RLS case management in the clinic, and this recording data was retrospectively obtained. The iron level was not measured at the follow-up evaluation, but in case of the recurrence of RLS symptoms during the follow-up period, the iron level test was done again to confirm whether the recurrence was related with iron deficiency. Sixteen patients were evaluated through face-to-face interview in the clinic and telephone interview was used for the rest of patients. We had asked the following question: "How are your RLS symptoms after iron normalization compared to those at the initial diagnosis?" Responses included 0) absent or negligible without any medication for RLS after stopping iron supplements, 1) similar, fluctuating, or deteriorating, and 2) taking some medications to control RLS symptoms. If the response was "0," the participant was assigned to the "remission" group. Those whose responses were "1" or "2" were assigned to the "persistence" group. The outcomes of this study were persistence of RLS symptoms and risk factors associated with RLS symptom persistence.

\section{Statistical analysis}

Between the remission and the persistence group, demo- 
graphic characteristics, clinical characteristics, and laboratory data were compared using chi-square test or student t-test. Mann-Whitney test was performed when data were not normally distributed. We used multivariable logistic regression analyses to estimate the relative risk for persisting RLS symptoms as a dependent variable. Odds ratios (OR) and 95\% confidence interval (CI) were determined after adjusting for the use of antidepressants, baseline serum ferritin level, family history and scores of BDI, PSQI and ESS as covariates. All tests of significance were two sided. p-value of less than 0.05 was considered as statistically significant. SPSS version 22.0 for Windows (IBM Corp., Armonk, NY, USA) was used in all analyses.

\section{RESULTS}

The study flow is shown in Figure 1. Among 61 patients who were diagnosed as RLS with iron deficiency, 8 patients droppedout in the process and 4 patients had underlying medical diseases (2 gastric cancer, 1 hypermenorrhea, 1 end stage renal disease). After excluding these 12 subjects, 49 patients of RLS were confirmed to have obtained normalization of serum iron and they stopped taking iron supplements. Among them, 10 patients ( 4 had no contact or refusal, 3 deceased, 1 rectal cancer, 2 gastric ulcer) were excluded. Finally, a total 39 RLS patients were included in our analyses. The mean age of the pa- tients at the first visit was $53.8 \pm 13.5$ (range, 14-76) years. Antidepressants were taken in 10 patients (paroxetine $=2$, venlafaxine $=2$, escitalopram $=2$, sertraline $=1$, mirtazapine $=1$, trazodone $=1$, nortriptyline $=1$ ) and 13 patients reported moderate to severe depressive symptoms according to BDI. Only two patients in the persistence group reported side effects including constipation and gastric discomfort. The demographics and RLS-related clinical characteristics at baseline were not significantly different between the drop-out subjects and the final participants.

Over the observation period of $2.5 \pm 1.4$ (range, $0.5-5.7$ ) years after iron normalization, only 15 patients reported no RLS symptom while 24 patients had persistent RLS symptoms or had been taking medication for RLS. Therefore, the remission rate was $38.5 \%$. Two patients in the remission group had reported the recurrence of RLS symptoms accompanied by low serum ferritin level at 3 and 6 months after the initial normalization. However, their symptoms were resolved with additional iron replacements which comprised the same dose and duration as in the initial treatment. Between the remission group and the persistence group, duration of RLS symptom was significantly different $(4 \pm 3.3$ years vs. $11.7 \pm 11.2$ years, $\mathrm{p}=0.004$ ). Age at RLS diagnosis also showed an increasing tendency in persistent group compared to remission group (48.7 \pm 14.8 years vs. $57 \pm 11.8$ years, $\mathrm{p}=0.059$ ). Although the mean score

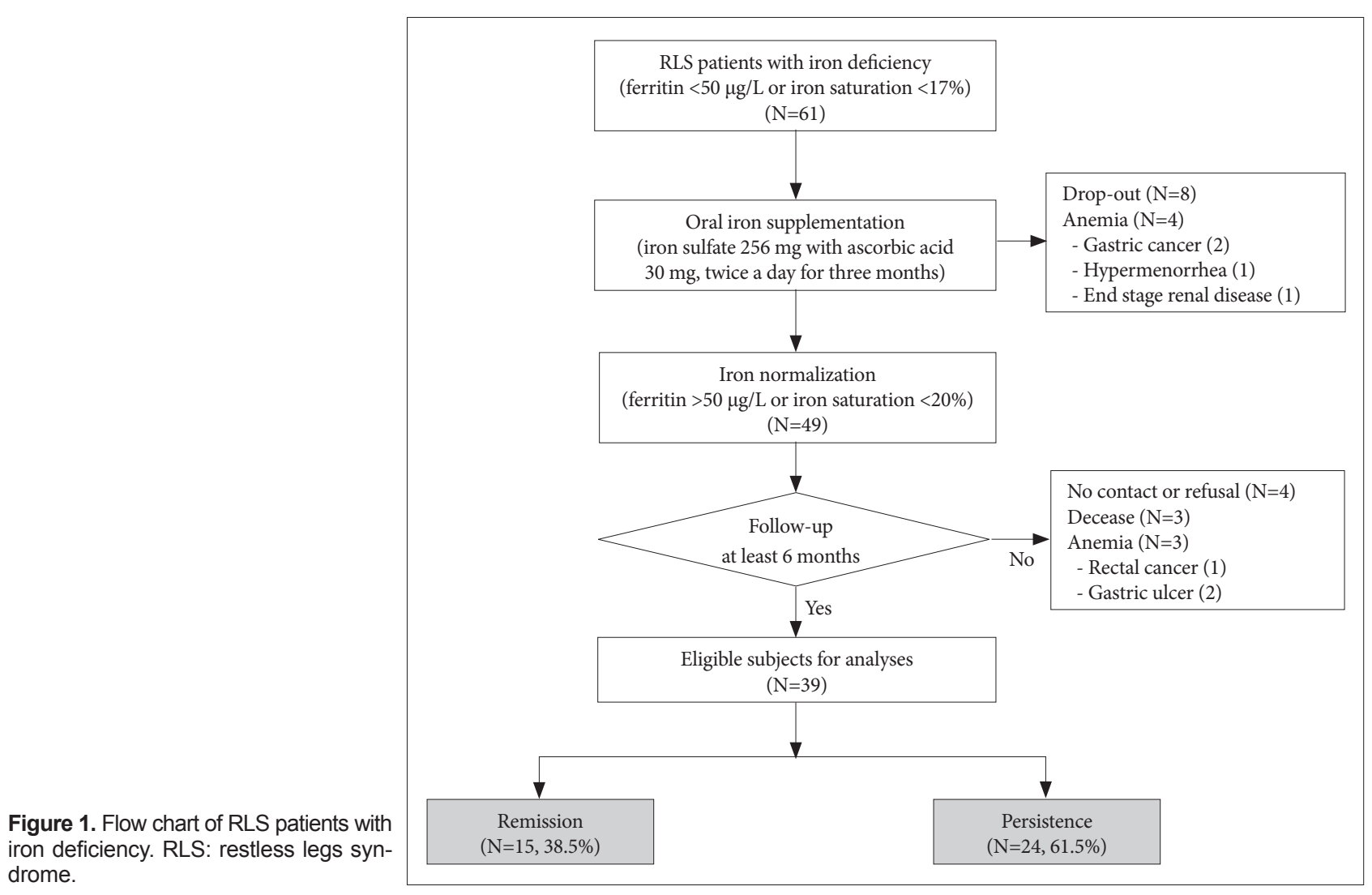


Table 1. Comparison of baseline demographics and clinical characteristics between the remission group and the persistence group

\begin{tabular}{|c|c|c|c|}
\hline & Remission $(\mathrm{N}=15)$ & Persistence $(\mathrm{N}=24)$ & p-value \\
\hline \multicolumn{4}{|l|}{ Demographics } \\
\hline Age at RLS diagnosis (year) & $48.67 \pm 14.82$ & $57.04 \pm 11.82$ & 0.059 \\
\hline Age at symptom onset (year) & $45.20 \pm 14.09$ & $45.29 \pm 18.73$ & 0.987 \\
\hline Duration of symptom (year) & $4.00 \pm 3.27$ & $11.69 \pm 11.21$ & 0.004 \\
\hline Duration of follow-up (year) & $3.11 \pm 1.86$ & $3.94 \pm 2.52$ & 0.274 \\
\hline$\geq 2$ multiparity & $10(76.9)$ & $19(79.2)$ & 0.874 \\
\hline \multicolumn{4}{|l|}{ Laboratory findings } \\
\hline Ferritin, baseline $(\mu \mathrm{g} / \mathrm{L})$ & $32.10 \pm 10.99$ & $27.05 \pm 11.26$ & 0.177 \\
\hline Ferritin, follow-up ( $\mu \mathrm{g} / \mathrm{L})$ & $83.02 \pm 47.49$ & $91.49 \pm 65.08$ & 0.665 \\
\hline Iron saturation, follow-up (\%) & $28.80 \pm 9.46$ & $30.48 \pm 7.72$ & 0.555 \\
\hline Changes of ferritin/month $(\mu \mathrm{g} / \mathrm{L})$ & $7.46 \pm 4.68$ & $9.90 \pm 7.75$ & 0.268 \\
\hline Hemoglobin (g/dL) & $13.16 \pm 0.81$ & $12.86 \pm 1.37$ & 0.509 \\
\hline \multicolumn{4}{|l|}{ Clinical features at baseline } \\
\hline IRLS scores & $24 \pm 8.73$ & $23.83 \pm 8.90$ & 0.955 \\
\hline RLS_severity (mild-moderate/severe-very severe) & $6(40) / 9(60)$ & $5(20.8) / 9(79.2)$ & 0.196 \\
\hline Symmetry (left/right/both)* & $4(30.8) / 4(30.8) / 5(38.5)$ & $5(20.8) / 6(25) / 13(54.2)$ & 0.646 \\
\hline Involvement of upper limb* & $2(15.4)$ & $3(12.5)$ & 1 \\
\hline Presence of pain* & $2(15.4)$ & $2(8.3)$ & 0.602 \\
\hline Family history* & $3(25)$ & $7(29.2)$ & 1 \\
\hline PSQI & $12.67 \pm 4.55$ & $12.27 \pm 4.63$ & 0.8 \\
\hline ESS & $7.6 \pm 4.27$ & $7.09 \pm 4.94$ & 0.747 \\
\hline BDI & $15.93 \pm 8.66$ & $13.05 \pm 8.76$ & 0.329 \\
\hline Antidepressant use (\%) & 26.7 & 25 & 0.908 \\
\hline
\end{tabular}

Data present number (percentage) and mean values \pm standard deviation (range). RLS severity based on the total IRLS score, mild (1-10), moderate (11-20), severe (21-30) and very severe (31-40). *Fisher's exact test. RLS: restless leg syndrome, IRLS: International RLS Study Group Scale for Severity, PSQI: pittsburgh sleep quality index, ESS: epworth sleepiness scale, BDI: Beck depression inventory

Table 2. Logistic regression analyses for the persistence of RLS symptom

\begin{tabular}{lccc}
\hline & OR & $95 \%$ CI & p-value \\
\hline Duration of RLS symptoms & 1.88 & $1.01-3.49$ & 0.048 \\
Age at RLS diagnosis & 1.25 & $1.01-1.56$ & 0.047 \\
RLS severity & 1.23 & $0.9-1.68$ & 0.191 \\
\hline
\end{tabular}

Confounding factors in this model were use of antidepressant, baseline serum ferritin level, family history, scores of BDI, PSQI and ESS. RLS severity based on the total IRLS score, mild (1-10), moderate (11-20), severe (21-30), and very severe (31-40). RLS: restless leg syndrome, BDI: beck depression inventory, PSQI: pittsburgh sleep quality index, ESS: epworth sleepiness scale, IRLS: International RLS Study Group Scale for Severity

of IRLS rating scale was similar to each other between the two groups, the persistence group showed a trend toward more severe RLS (79.2\%) than those in the response group (60\%) when RLS severity was classified into mild to moderate and severe to very severe. Other demographic characteristics, serum ferritin level and RLS-related clinical features including anti- depressant use were not significantly different between the two groups (Table 1).

Multivariable logistic regression analyses showed that RLS symptom duration at baseline and the age at RLS diagnosis were significantly associated with the persistence of RLS symptom after iron normalization (Table 2). There was no multicollinearity between symptom duration and age at diagnosis (Pearson's coefficient of determinant $=-0.075, \mathrm{p}=0.639$ ). Long duration of RLS symptom significantly increased the relative risk of symptom persistence (OR: 1.88, 95\% CI: 1.01-3.49, p= 0.048). In addition, the age at RLS diagnosis was significantly associated with symptom persistence. The relative risk of symptom persistence was higher when the age at diagnosis was older (OR: 1.25, 95\% CI: 1.01-1.56, p=0.047). However, RLS severity was not significantly associated with the symptom persistence.

\section{DISCUSSION}

This study for the first time investigated the clinical course 
and persistence of RLS symptoms after iron replacement treatment in secondary RLS with low serum ferritin levels. The main findings were that almost two-third patients of RLS with iron deficiency showed the persistence of RLS symptoms even after iron normalization. In addition, longer duration of RLS symptom and older age at RLS diagnosis contributed to RLS symptom persistence.

The remission rate of iron treatment was found to be $38.5 \%$ in this study, which was slightly lower than $46.7 \%$ of our previous study after comparing the efficacy of treatments between oral iron and pramipexole in RLS patients with low serum ferritin. ${ }^{9}$ The discrepancy might be due to different definitions used for treatment efficacy. In this study, we examined a relatively strict outcome of the remission state defined as no or negligible symptom. In our previous study, response state was defined as symptom reduction of 50\% after oral iron treatment. Thus, the overall remission rate of oral iron treatment could be estimated to be around $40 \%$. The remission rate of $38.5 \%$ in this study was unexpected because substantial RLS symptom control was supposed to be achieved after serum ferritin level became $50 \mu \mathrm{g} / \mathrm{L}$ or more. As secondary RLS with iron deficiency was defined as serum ferritin level of less than $50 \mu \mathrm{g} / \mathrm{L}$ or iron saturation of less than $17 \%$, the improvement of RLS symptom would have been reasonably predicted with iron normalization of $\geq 50 \mu \mathrm{g} / \mathrm{L}$. The reason for relatively low remission rate after iron normalization might be due to the fact that many cases of secondary RLS with iron deficiency had the pathophysiology of primary RLS at the same time. Among possible pathophysiology of primary RLS, brain iron deficiency and central dopamine dysfunction have been suggested. Serum ferritin level has been accepted as the best single measurement of iron store. ${ }^{14}$ However, it is not an indicative marker of brain iron stores. Peripheral iron concentrations have no linear association with brain iron concentrations. ${ }^{7}$ For this reason, regional brain iron deficiency still could have contributed to an abnormality of dopaminergic system that produces symptoms of RLS, even though the peripheral iron status has been normalized. ${ }^{15-18}$ To control RLS symptoms, IV iron treatment is known to work better by maintaining higher levels of iron availability for short periods and by providing rich and fast iron supply to the brain. RLS symptoms might persist since oral iron supplement could not be effective enough to penetrate brain-blood barriers or replenish iron into brain regions as shown in the present study. The higher response rate (65\%) with IV iron injections in previous experimental study with secondary RLS ${ }^{7}$ might be due to pathophysiological correction of primary RLS and secondary RLS. To examine the effect of IV iron treatments on RLS, most controlled trials have been carried out for patients with normal iron store of $\geq 50 \mu \mathrm{g} / \mathrm{L}$ of serum ferritin levels. ${ }^{19-21}$ Those trials might have targeted primary RLS rather than secondary RLS.

In this study, patients who had longer duration of RLS showed a higher risk of symptom persistence. Although we could not argue that the duration of RLS symptom and that of iron deficiency were the same, we might cautiously assume an association between both durations. Indeed, a close relationship between the decrease of serum ferritin level and the occurrence of RLS symptom has been reported in some RLS patients who had a tendency to rapidly deplete iron stores. ${ }^{15}$ It has been reported that repeated experiences of RLS caused by multi-parity are strongly associated with increasing incidence of RLS not related to pregnancy, ${ }^{22}$ suggesting that repeated and chronic exposure of peripheral iron deficiency due to pregnancies might cause cumulative pathophysiologic changes on overall iron metabolism including brain, regardless of peripheral iron deficiency. Thus, longer duration of RLS in line with longer state of iron deficiency might have contributed to the development of primary RLS which was not improved with peripheral iron normalization in the present study. Another mechanism of disease duration-related RLS persistence could be associated with neuronal damages such as chronic hypoxia and myelin loss. These neuronal damages can be caused by brain iron deficiency because oxygen transport and myelin synthesis demand proteins, lipids, and cholesterol, all of which are iron dependent. ${ }^{23}$ Therefore, it is important to notice that longer duration of RLS symptoms is a potential risk factor for RLS symptom persistence. Early intervention for RLS can provide a better chance for symptom improvement.

Older age at RLS diagnosis was another risk factor for RLS symptom persistence after iron normalization. In women, it has been found that serum ferritin levels remain low from adolescent until the menopause because of menstrual cycles and pregnancy. They gradually rise to levels comparable to those in males after menopause. ${ }^{24}$ Meanwhile, the occurrence of RLS in elderly individuals is well known. ${ }^{25}$ RLS prevalence increases with age. It reaches its peak around fifties in both genders with a clear tendency in women, then it remains as a plateau. ${ }^{26}$ Thus, peripheral iron deficiency in middle aged to old RLS patients of this study might be an incidental finding rather than main causes of RLS. These patients might have persistent symptoms even after correction of peripheral iron deficiency.

When we observed the clinical course of RLS patients for at least 6 months or longer, the incidence of recurrence associated with iron deficiency was reported in two cases. The pattern of recurrence caused by recurrent iron deficiency has been reported in a previous study of Grote et al. ${ }^{7}$ We found that RLS symptoms reappeared at 3 and 6 months after the initial oral iron treatment. Early et al. ${ }^{27}$ have reported that five 
cases need to repeat IV iron injection due to recurred symptoms at an average of 6 months (range, 3 to 13 months) after the initial injection. It is currently unclear how long iron treatment needs to be provided to RLS patients with low serum ferritin levels or when iron deficiency will recur. RLS patients with iron deficiency may stop taking oral or IV iron after iron normalization and symptom improvement. They need to be regularly followed up to determine the recurrence of RLS symptom and iron deficiency.

This study has several limitations. First, a recall bias regarding symptom duration might have occurred because the data of onset age were collected depending on participants' memory. The recall bias is, to some extent, unavoidable in retrospective observational studies for a long duration. Second, no information about IRLS scores was available at the follow-up interview. It was difficult to obtain IRLS scores from telephone interview, and many patients in the persistent group already took RLS medications, making it difficult to interpret the meaning of IRLS scores. Third, the range of follow-up period was too large (from 1 to 6 years). Participants with relatively short duration in the remission group could develop RLS symptoms in the near future. Nevertheless, this study provided valuable information about the clinical course of RLS patients with low serum ferritin levels after iron normalization. In secondary RLS with iron deficiency, the efficacy of oral iron replacement treatment was rather limited. Developing better methods for iron treatment and considering alternative treatment will be needed to control persistent RLS symptoms even after iron normalization. We found that longer duration of symptoms and older age at RLS diagnosis decreased the treatment effect of oral iron but increased the risk of symptom persistence. Therefore, this study provides clinical implication that early intervention of iron deficiency in RLS is needed.

\section{REFERENCES}

1. Kushida CA. Clinical presentation, diagnosis, and quality of life issues in restless legs syndrome. Am J Med 2007;120(1 Suppl 1):S4-S12.

2. Lee CS, Kim T, Lee S, Jeon HJ, Bang YR, Yoon IY. Symptom severity of restless legs syndrome predicts its clinical course. Am J Med 2016;129: 438-445.

3. Allen R. Dopamine and iron in the pathophysiology of restless legs syndrome (RLS). Sleep Med 2004;5:385-391.

4. Silber MH, Ehrenberg BL, Allen RP, Buchfuhrer MJ, Earley CJ, Hening WA, et al. An algorithm for the management of restless legs syndrome. Mayo Clinic Proc 2004;79:916-922.

5. Thorpy MJ. New paradigms in the treatment of restless legs syndrome. Neurology 2005;64(12 Suppl 3):S28-S33.

6. Wang J, O'Reilly B, Venkataraman R, Mysliwiec V, Mysliwiec A. Efficacy of oral iron in patients with restless legs syndrome and a low-normal ferritin: a randomized, double-blind, placebo-controlled study. Sleep Med 2009;10:973-975.

7. Grote L, Leissner L, Hedner J, Ulfberg J. A randomized, double-blind, placebo controlled, multi-center study of intravenous iron sucrose and placebo in the treatment of restless legs syndrome. Mov Disord 2009;24:
1445-1452.

8. Walters AS, LeBrocq C, Dhar A, Hening W, Rosen R, Allen RP, et al. Validation of the International Restless Legs Syndrome Study Group rating scale for restless legs syndrome. Sleep Med 2003;4:121-132.

9. Lee CS, Lee SD, Kang SH, Park HY, Yoon IY. Comparison of the efficacies of oral iron and pramipexole for the treatment of restless legs syndrome patients with low serum ferritin. Eur J Neurol 2014;21:260-266.

10. Allen RP, Picchietti D, Hening WA, Trenkwalder C, Walters AS, Montplaisi J, et al. Restless legs syndrome: diagnostic criteria, special considerations, and epidemiology. A report from the restless legs syndrome diagnosis and epidemiology workshop at the National Institutes of Health. Sleep Med 2003;4:101-119.

11. Allen RP, Picchietti DL, Garcia-Borreguero D, Ondo WG, Walters AS, Winkelman JW, et al. Restless legs syndrome/Willis-Ekbom disease diagnostic criteria: updated International Restless Legs Syndrome Study Group (IRLSSG) consensus criteria--history, rationale, description, and significance. Sleep Med 2014;15:860-873.

12. Buysse DJ, Reynolds Iii CF, Monk TH, Berman SR, Kupfer DJ. The Pittsburgh sleep quality index: a new instrument for psychiatric practice and research. Psychiatry Res 1989;28:193-213.

13. Johns MW. A new method for measuring daytime sleepiness: the Epworth sleepiness scale. Sleep 1991;14:540-545.

14. Alvarez-Ossorio L, Kirchner H, Kluter H, Schlenke P. Low ferritin levels indicate the need for iron supplementation: strategy to minimize iron-depletion in regular blood donors. Transfus Med 2000;10:107-112.

15. Allen RP, Earley CJ. The role of iron in restless legs syndrome. Mov Disord 2007;22(Suppl 18):S440-S448.

16. Earley CJ, Connor JR, Beard JL, Malecki EA, Epstein DK, Allen RP. Abnormalities in CSF concentrations of ferritin and transferrin in restless legs syndrome. Neurology 2000;54:1698-1700.

17. Connor JR, Wang XS, Patton SM, Menzies SL, Troncoso JC, Earley CJ, et al. Decreased transferrin receptor expression by neuromelanin cells in restless legs syndrome. Neurology 2004;62:1563-1567.

18. Connor JR, Ponnuru P, Wang XS, Patton SM, Allen RP, Earley CJ. Profile of altered brain iron acquisition in restless legs syndrome. Brain 2011;134:959-968.

19. Davis BJ, Rajput A, Rajput ML, Aul EA, Eichhorn GR. A randomized, double-blind placebo-controlled trial of iron in restless legs syndrome. Eur Neurol 2000;43:70-75.

20. Sloand JA, Shelly MA, Feigin A, Bernstein P, Monk RD. A double-blind, placebo-controlled trial of intravenous iron dextran therapy in patients with ESRD and restless legs syndrome. Am J Kidney Dis 2004;43:663670.

21. Earley CJ, Horska A, Mohamed MA, Barker PB, Beard JL, Allen RP. A randomized, double-blind, placebo-controlled trial of intravenous iron sucrose in restless legs syndrome. Sleep Med 2009;10:206-211.

22. Ru Y, Pressman EK, Cooper EM, Guillet R, Katzman PJ, Kent TR, et al. Iron deficiency and anemia are prevalent in women with multiple gestations. Am J Clin Nutr 2016;104:1052-1060.

23. Allen RP. Restless Leg Syndrome/Willis-Ekbom Disease Pathophysiology. Sleep Med Clin 2015;10:207-214.

24. Milman N. Serum ferritin in Danes: studies of iron status from infancy to old age, during blood donation and pregnancy. Int J Hematol 1996;63: 103-135.

25. Kim KW, Yoon IY, Chung S, Shin YK, Lee SB, Choi EA, et al. Prevalence, comorbidities and risk factors of restless legs syndrome in the Korean elderly population-results from the Korean Longitudinal Study on Health and Aging. J Sleep Res 2010;19:87-92.

26. Nichols DA, Allen RP, Grauke JH, Brown JB, Rice ML, Hyde PR, et al. Restless legs syndrome symptoms in primary care: a prevalence study. Arch Intern Med 2003;163:2323-2329.

27. Earley CJ, Heckler D, Allen RP. Repeated IV doses of iron provides effective supplemental treatment of restless legs syndrome. Sleep Med 2005;6:301-305. 